\title{
Mucormycosis: Changing Profile - Analytical study of cases admitted to tertiary care hospital
}

\author{
Authors \\ Dr Soham Kadam ${ }^{1}$, Dr Jitendra Ingole ${ }^{2}$, Dr Dileep Kadam ${ }^{3}$, Dr Shreepad Bhat ${ }^{4}$ \\ ${ }^{1}$ Post Graduate Student in Internal Medicine, Department of Internal Medicine, Smt Kashibai Navale Medical College, \\ Pune \\ ${ }^{2,3}$ Professor and Head of Unit, Department of Internal Medicine, Smt Kashibai Navale Medical College, Pune \\ ${ }^{4}$ Professor and Head of Department, Department of Internal Medicine, Smt Kashibai Navale Medical College, Pune
}

\section{Introduction}

Mucormycosis is one of the most underrated disease of present age. Due to rise in number of cases of Diabetes Mellitus, use of immunosuppressive medications, cases of Mucormycosis are on a rise. The disease usually presents with docile symptoms to start of with and is caught at a severe stage

Mucormycosis is caused by a fulminating fungus caused by members of the Mucoraceae, order Mucorales and class Zygomycetes. These are ubiquitous fungi growing primarily on decaying vegetation and organic material. It was first described by Paulltauf in 1885. Most of the patients infected have an underlying predisposing condition favouring the growth of the organism.

Rhizopus organism possess an enzyme, ketone reductase what allow them to flourish in high glucose, acidic conditions.

Most common sites for Mucormycosis infection are sinuses leading to a spectrum of diseases from Sino-nasal, Rhino-Orbital and Rhino-OrbitoCerebral. Other sites of infection include Pulmonary Mucormycosis, Gastro-Intestinal Mucormycosis, Cutaneous Mucormycosis, Renal Mucormycosis, Isolated CNS Mucormycosis,
Disseminated Disease. Mode of Spread is Primarily Angioinvasive followed by Locoregional Spread in Sinus infections, where in the pterygopalatine fossa acts as a resiorviour for the fungus.

Almost all the patients of this disease has an underlying condition most common have been listed below

- Diabetes mellitus, particularly with ketoacidosis

- Treatment with glucocorticoids and immunosuppressive drugs

- Malnutrition

- transplantation patients

- AIDS

- Iron overload

- Trauma/burns

\section{Material and Method}

All consecutive Cases of Mucormycosis admitted between the time periods of 1 year 26 JUNE 2017- 26 JUNE 2018, Admitted in Internal Medicine and Otto Rhyno Laryngology wards were studied for their entire course of hospital stay. 
Inclusion Criteria: 1.All patient with Radiological and/or Histopathological with appropriate medical history pointing to mucormycosis were included in this study. The patients were studied for disease presentation and progression, medication related complications and overall survival rate.

Functional Endoscopic Sinus Surgery was done in all patients that could tolerate the procedure based on general condition and lab parameters.

Radiological imaging (CT PNS) done in all patients and were graded as per the Lund-Mackey grading system for sinusitis.

Patients were evaluated for glycemic control, Acute Kidney Injury due to Medication, Changes in other lab parameters that could affect patient outcome. Histopathological conformation was done using Special stands namely Periodic Acid Schiff (PAS) and Silver Methenamine stain.

\section{Exclusion Criteria: Nil}

\section{Statistical Analysis}

The statistical values were tabulated and appropriate statistical tests were applied for evaluation of data

\section{Results}

A total of 10 patients were studied who fit the inclusion criteria

1. Age Vs Sex

\begin{tabular}{|l|c|c|}
\hline Age & MALE & FEMALE \\
\hline$<20$ & 0 & 0 \\
\hline $\mathbf{2 1 - 3 0}$ & 0 & 0 \\
\hline $\mathbf{3 1 - 4 0}$ & 1 & 0 \\
$\mathbf{4 1 - 5 0}$ & 0 & 1 \\
\cline { 2 - 3 } $\mathbf{5 1 - 6 0}$ & 1 & 2 \\
\hline $\mathbf{6 1 - 7 0}$ & 4 & 0 \\
\hline $\mathbf{7 1 - 8 0}$ & 0 & 0 \\
\hline
\end{tabular}

2. Predisposing Conditions

\begin{tabular}{|c|c|}
\hline Condition & Number \\
\hline DIABETE MELLITUS & 9 \\
\hline HYPERTENSION & 3 \\
\cline { 2 - 3 } CVA with ryles tube in situ & 1 \\
\hline RA ON MEDICATIONS & 1 \\
\hline SINUSITIS & 1 \\
\hline ASTHAMA & 0 \\
\hline
\end{tabular}

3. Diabetes Since

\begin{tabular}{|l|c|}
\hline Diabetes since & Number \\
\hline$<1$ MONTH & 5 \\
\hline 1 MONTH TO 1 YEAR & 1 \\
\cline { 2 - 2 }$>$ 1 YEAR & 3 \\
\hline
\end{tabular}

4. Presenting Complaints

\begin{tabular}{|l|c|}
\hline Presenting complaints & Number \\
\hline SINUSITIS & 7 \\
\hline HEADACHE & 7 \\
\hline FACIAL SWELLING & 6 \\
\hline NASAL DISCHARGE & 6 \\
\hline DECREASED VISION & 5 \\
\hline FEVER & 4 \\
\hline LOSS OF POWER & 3 \\
\hline OPTHALMOPLEGIA & 2 \\
\hline ORAL ULCERS/ABSCESSES & 2 \\
\hline NASAL ULCERATION & 1 \\
\hline
\end{tabular}

5. Glassglow Coma Scale

\begin{tabular}{|c|c|c|c|}
\hline & & \multicolumn{2}{|c|}{ OUTCOME } \\
\hline & & DISCHARGED & EXPIRED \\
$\mathbf{1 3 - 1 5}$ & 8 & 8 & 0 \\
\hline $\mathbf{9 - 1 2}$ & 1 & 0 & 1 \\
\hline $\mathbf{3 - 8}$ & 1 & 0 & 1 \\
\hline
\end{tabular}


6. Cranial Nerves Involved

\begin{tabular}{|l|l|}
\hline \multirow{2}{*}{ II } & 1 \\
\hline III & 3 \\
\cline { 2 - 2 } IV & 3 \\
\hline V & 1 \\
\hline VI & 4 \\
\hline VII & 2 \\
\hline
\end{tabular}

Rest of the cranial nerves were found normal

7. Blood Sugar Levels

\begin{tabular}{|l|c|c|}
\hline & & \\
\hline$<200$ & 1 & 0 \\
\hline $\mathbf{2 0 0 - 3 0 0}$ & 5 & 0 \\
\hline $\mathbf{3 0 0 - 4 0 0}$ & 1 & 0 \\
\hline$>\mathbf{4 0 0}$ & 3 & 1 \\
\hline
\end{tabular}

\section{8. $\mathrm{HbA1c}$}

\begin{tabular}{|l|c|c|c|}
\hline & & \multicolumn{2}{|c|}{ OUTCOME } \\
\hline & & DISCHARGED & EXPIRED \\
\hline$<\mathbf{5 . 7}$ & 1 & 0 & 1 \\
\hline $\mathbf{5 . 7 - 6 . 4}$ & 1 & 1 & 0 \\
\hline$>\mathbf{6 . 4}$ & 8 & 7 & 1 \\
\hline
\end{tabular}

9. Lund-Mackey grading system for CT PNS findings

\begin{tabular}{|l|c|c|c|}
\hline & & \multicolumn{2}{|c|}{ OUTCOME } \\
\hline & & DISCHARGED & EXPIRED \\
\hline $\mathbf{0 - 5}$ & 2 & 1 & 1 \\
\cline { 2 - 4 } $\mathbf{5 - 1 0}$ & 4 & 3 & 1 \\
$\mathbf{3 1 0}$ & 4 & 4 & 0 \\
\hline
\end{tabular}

10. FESS with Surgical Debridement and Histological conformation

\begin{tabular}{|l|c|c|c|}
\hline & & \multicolumn{2}{|c|}{ OUTCOME } \\
\hline & & DISCHARGED & EXPIRED \\
\hline DONE & 7 & 7 & 0 \\
\hline NOT DONE & 3 & 1 & 2 \\
\hline
\end{tabular}

11.As per classification

\begin{tabular}{|l|c|c|c|}
\hline & & \multicolumn{2}{|c|}{ OUTCOME } \\
\hline & & DISCHARGED & EXPIRED \\
\hline SINO-NASAL & 4 & 4 & 0 \\
\hline RHYNO-ORBITAL & 1 & 1 & 0 \\
\hline $\begin{array}{l}\text { RHYNO-ORBITO- } \\
\text { CEREBRAL }\end{array}$ & 5 & 3 & 2 \\
\hline
\end{tabular}

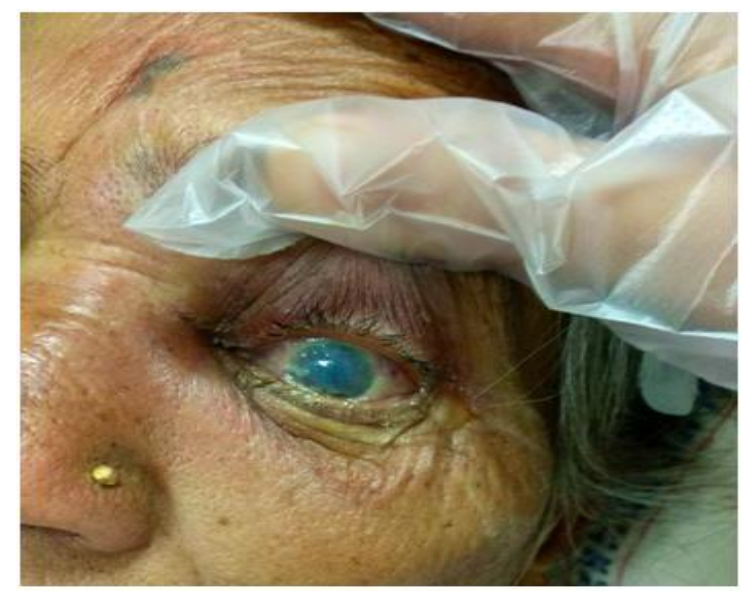

1a. orbital involvement in rhyno-orbito-cerebral mucormycosis

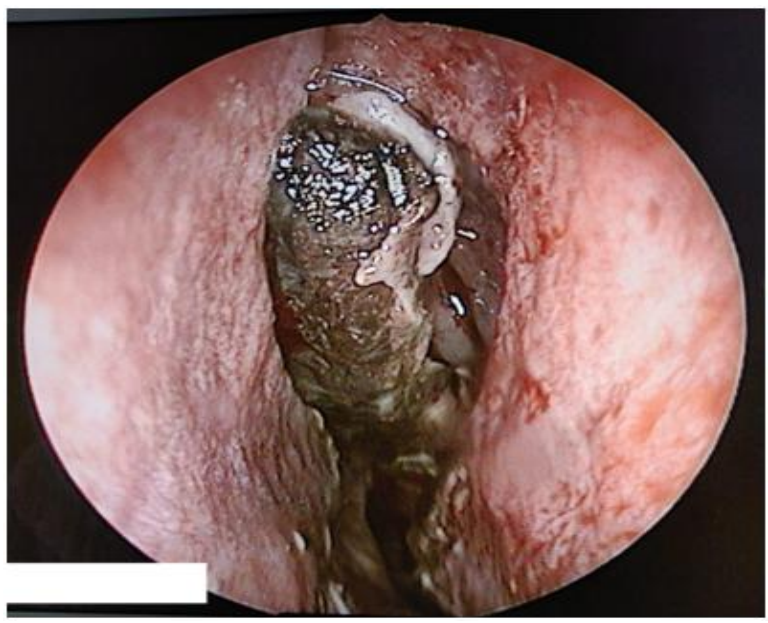

1.b FESS image suggestive of Black Homegenous mass 


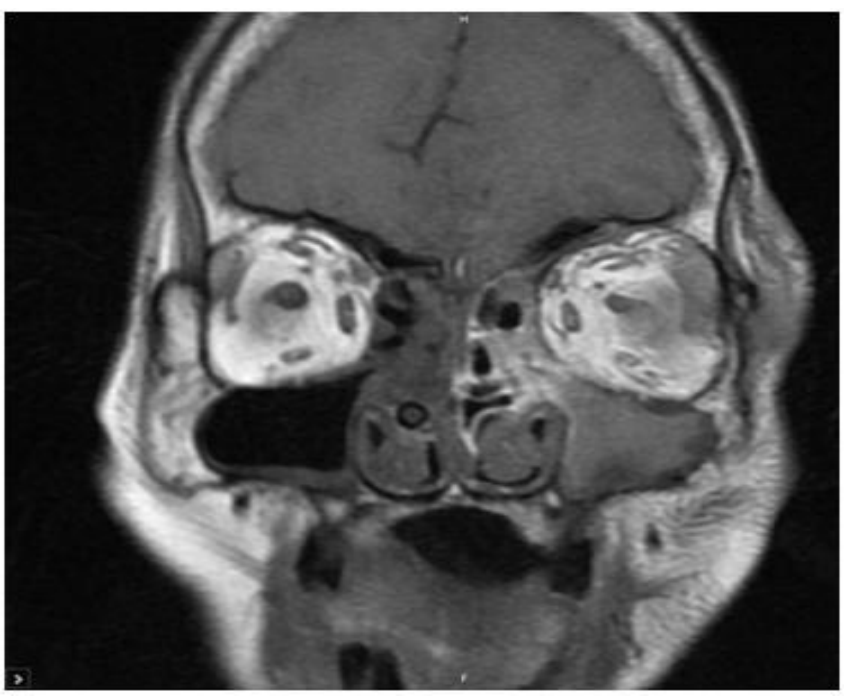

1c. Radiological Image of PNS with sinus fullness

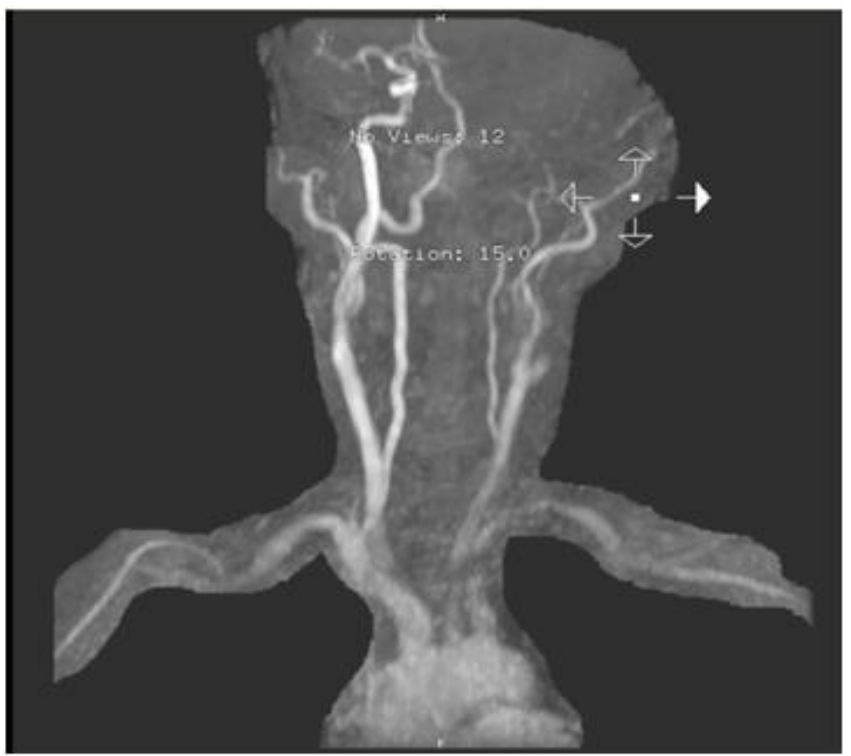

1d. MR Angio suggestive of internal carotid obstruction in a patient, patient expired.

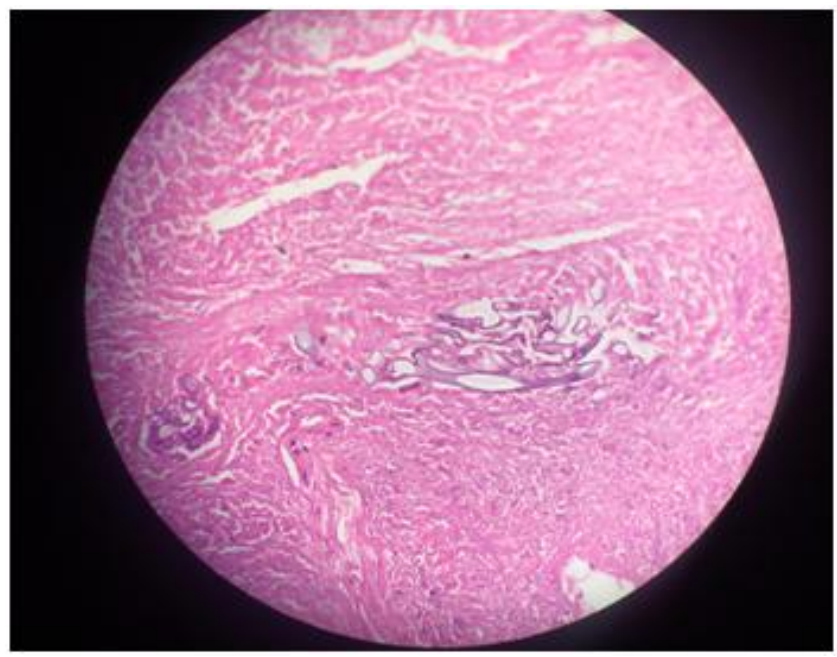

Image 2a- Histopathological examination with $\mathrm{H}$ and $\mathrm{E}$ stain demonstrating Mucormycosis.

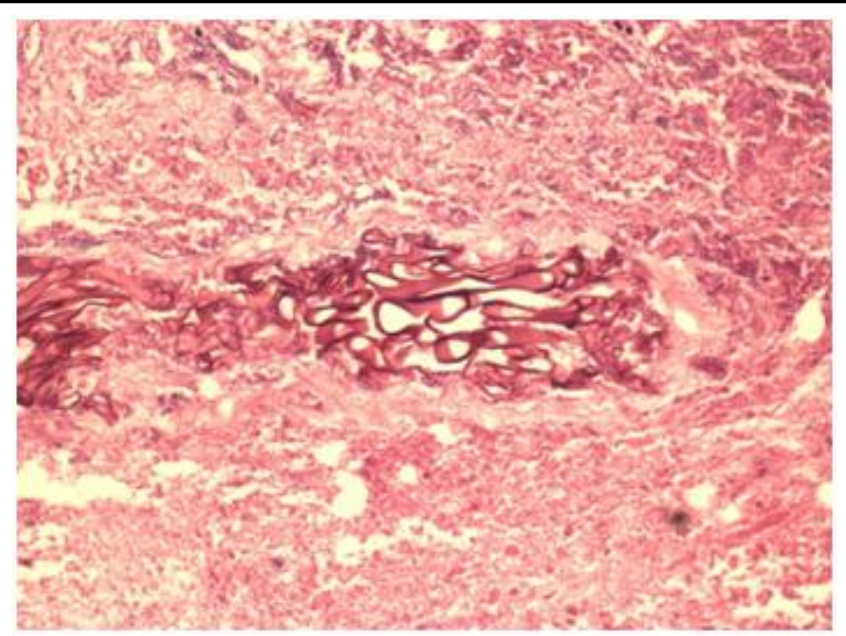

2b. PAS staining

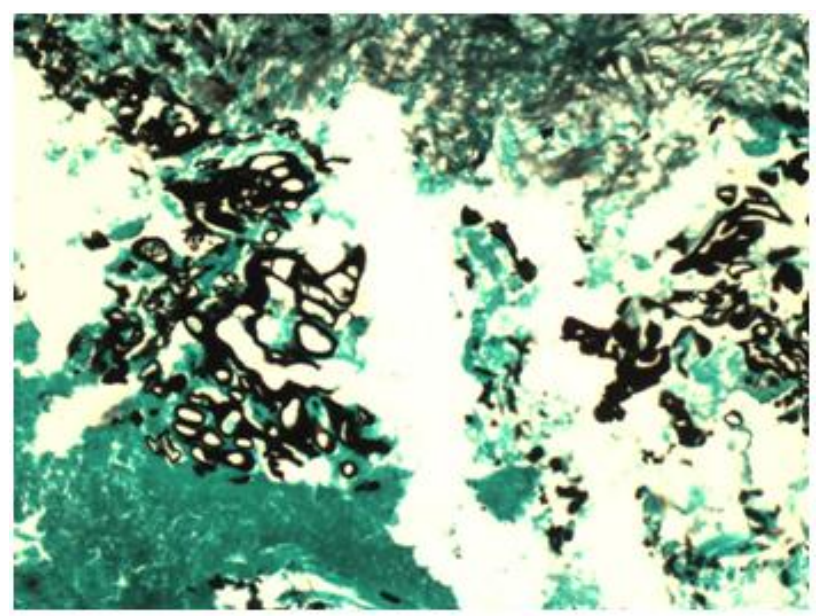

2c. Silver Methamine staining

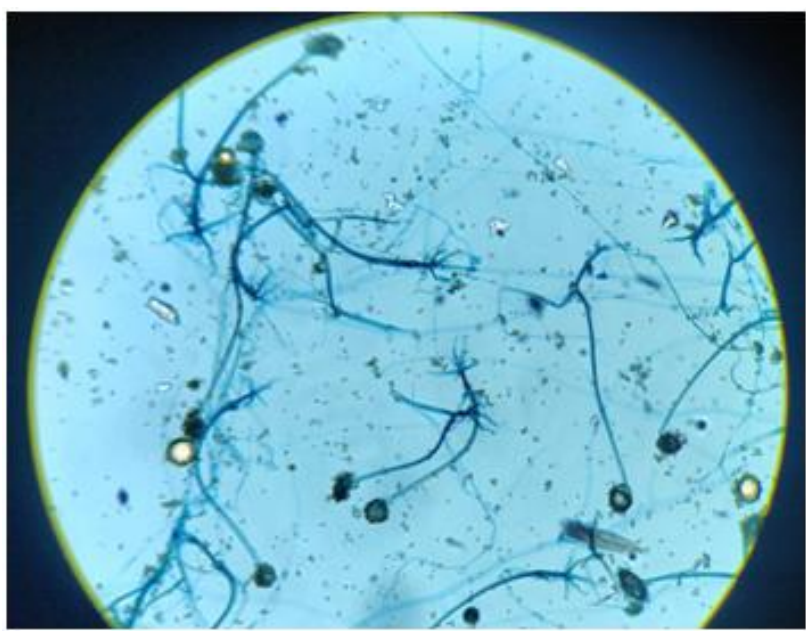

2d. microscopic conformation of species as RhizopusOryzae

\section{Discussion}

In 1 year study period 10 case were identified suggesting an increase in Rhizopus infections. Most of the patients were from a lower socio- 
economic strata. The most frequently isolated species for Mucormycosis is Rhizopus Oryzae followed by Rhizopus microsporus, and Absidia corymbifera in order of occurrence.I $\mathrm{n}$ our study Culture positivity was noted in 1 patient and was confirmed to Rhizopus Oryzae.

Mean age found to be 56.3 differing form the findings of Roden MM et al who noted a mean one of 38.8, whereas incidence in males being $70 \%$ similar to their study noting $65 \% .^{1}$

Competing to the study done by Yohai RA et al the following change in presenting symptoms was noted $^{2}$

\begin{tabular}{|l|c|c|}
\hline $\begin{array}{l}\text { Presenting } \\
\text { complaints }\end{array}$ & $\begin{array}{c}\text { Our Study(in } \\
\text { percentage) }\end{array}$ & $\begin{array}{c}\text { Yohai RA et al } \\
\text { study( in percentage) }\end{array}$ \\
\hline SINUSITIS & 70 & 26 \\
HEADACHE & 70 & 25 \\
\hline $\begin{array}{l}\text { FACIAL } \\
\text { SWELLING }\end{array}$ & 60 & 34 \\
\hline $\begin{array}{l}\text { NASAL } \\
\text { DISCHARGE }\end{array}$ & 60 & \\
\hline $\begin{array}{l}\text { DECREASED } \\
\text { VISION }\end{array}$ & 50 & 30 \\
\hline FEVER & 40 & 44 \\
\hline $\begin{array}{l}\text { LOSS } \\
\text { POWER }\end{array}$ & 30 & \\
\hline $\begin{array}{l}\text { OPTHALMOPLE } \\
\text { GIA }\end{array}$ & 20 & \\
\hline $\begin{array}{l}\text { ORAL } \\
\text { ULCERS/ABSCE } \\
\text { SSES }\end{array}$ & 20 & \\
\hline $\begin{array}{l}\text { NASAL } \\
\text { ULCERATION }\end{array}$ & 10 & \\
\hline
\end{tabular}

$80 \%$ of the patients presented to the OPD with a GCS above 13, with $10 \%$ with a GCS $<13$ and $10 \%$ with GCS <8. $50 \%$ presented with Cranial Nerve involvement, Most commonly the $\mathrm{V}^{\text {th }}$ nerve followed by II $^{\text {nd }}$ III $^{\text {rd }}$ and Its with $30 \%$ having hemiparesis.

Sinus Tenderness was noted in $90 \%$ of the patients, with $55.5 \%$ having bilateral sinus tenderness. Poor oral hygiene was noted in 60\%(6 patients) of the patient with $10 \%$ having tooth abscesses, and 30\% displaying Nasal ulcerations and crusting.

Comparing the lab parameters, $80 \%$ had a normal haemoglobin with $10 \%$ displaying polycythemia with superadded Diabetes Mellitus. 30\% had a total Leucocyte count more than 15000 suggesting towards sepsis and possibility of a disseminated Mucormycosis.

Renal parameters were consistent with $30 \%$ presenting with Acute Kidney Disease, 40\% landing up in Acute Kidney Disease on starting therapy with Amphoterecin B, which later improved on completing course of treatment. AKI was noted in all the patient who expired. Amphoterecin B is expected to cause hypokalema, in our study where a drop in potassium level as compared to admission was noted in only $20 \%$ (2 patients) of the patients.

Electrolyte imbalances were noted in majority of the patients with $50 \%$ presenting with Hyponatremia, 30\% with Hypokalemia, 10\% with hyperkalemia, and 20\%with hypochloremia. Comparing to studies abroad ${ }^{3}$ who noted Diabetes mellitus, particularly with ketoacidosis to be the most common predisposing factor, Indian studies also showed higher incidence in Diabetics ${ }^{5,6}$, our study displayed a similar result with $90 \%$ of the patients with Diabetes Mellitus, $40 \%$ with BSL more than 300 , and $10 \%$ in ketoacidosis. Control of Diabetes is a most important variable in outcome of Mucormycosis. $90 \%$ of the patient were Diabetics (old and newly diagnosed) with $50 \%$ of the patients presented with BSL of $>200$, $10 \%$ with BSL of $>300$ and $30 \%$ with BSL $>400$. The mean HbA1C was found to be 10.26 with a highest being $15.0 .10 \%$ of these patients were reported with Ketoacidosis.

On radioimaging with CT Scan of Paranasal Sinuses with a mean Lund-Mackay Score of 8.2. Lund-Mackay score was found to be a poor prognostic marker for Mucormycosis with Death noted with a score of 4 and 10, whereas patients with a score of 12 improved and were discharged. MRI brain done in patients with loss of power 
pointed to acute to subacute infarct, with one patient showing complete thrombosis of internal carotid artery.

Functional Endoscopic Sinus Surgery with debridement was carried out in $70 \%$ of the patients who were found fit to undergo the procedure. Samples from these were histopathologially confirmed to be Mucormycosis. Treatment was started early on the basis of Clinical Suspicion and Radiological investigations supporting the diagnosis. All Patients were given Amphoterecin B 1.0-1.5 mg/kg/day with dose adjustments in patients who developed complication (40\% developed Acute Kidney Injury on starting therapy). $30 \%$ were started on amphotericin B nasal drops. 30\% patients were started on dual antifungal therapy with either Fluconazole or Itraconazole. $10 \%$ of the patents received intra sinus fluconazole therapy. $60 \%$ patients were taken up for surgical debridement, and $10 \%$ required enucleation and destructive surgery. $40 \%$ of the patients were given antibiotic coverage. All patients receiving FESS with debridement had a better survival rate $100 \%$ compared to those who did not undergo debridement.

Other comparable studies ${ }^{3,4}$ noted a survival rate of $61 \%$ in patients treated with amphotericin B, comparing it to our study with a survival rate of $80 \%$ and a mortality of $20 \%$

On comparing the outcome $40 \%$ of the patients recovered completely, $40 \%$ had residual neurodeficit and $20 \%$ expired.

\section{Conclusion}

Cases of Mucormycosis are on a rise contributing to increasing cases of Diabetes Mellitus in absence of ketoacidosis, use of immunomodulator therapy in rheumatic disorders, owing to a decrease in defense mechanism against this disease. A diagnosis of this disease should be kept in bind while treating patients with minor complains of rhinorhea, sinus tenderness, headache with an underlying immunosuppressed condition. Early and prompt treatment should be started without awaiting histopathological conformation based on clinical and radiological evidence, this can intern lead to decrease mortality. Dose adjustments should be taken care of and close monitoring should be done in patients on treatment. Hypokalemia (20\%) and Acute Kidney Injury (40\%) with Amphoterecin B therapy was noted. Mortality rate was $20 \%$ seen in disseminated mucormycosis and rhino cerebral involvement.

\section{References}

1. Roden MM, Zaoutis TE, Buchanan WL, et al. Epidemiology and outcome of zygomycosis: a review of 929 reported cases. Clin Infect Dis 2005; 41:634.

2. Yohai RA, Bullock JD, Aziz AA, Markert RJ. Survival factors in rhino-orbitalcerebral mucormycosis. Surv Ophthalmol 1994; 39:3.

3. Roden MM, Zaoutis TE, Buchanan WL, et al. Epidemiology and outcome of zygomycosis: a review of 929 reported cases. Clin Infect Dis 2005; 41:634.

4. A Ghafur, PS Shareek, Nambi P Senthur, PR Vidyalakshmi, V Ramasubramanian, Ashok Parameswaran, MA Thirunarayan, $\mathrm{R}$ Gopalakrishnan. Mucormycosis in Patients without Cancer: A Case Series from A Tertiary Care Hospital in South India. Journal of the association of physicians of India2013; 61:11

5. A. Chakrabartietal. The rising trend of invasive zygomycosis in patients with uncontrolled diabetes mellitus. Med Mycol 2006;44:335-42. 